\title{
Endoscopic ultrasound-guided jejuno-jejunal anastomosis for malignant outlet obstruction after total gastrectomy
}

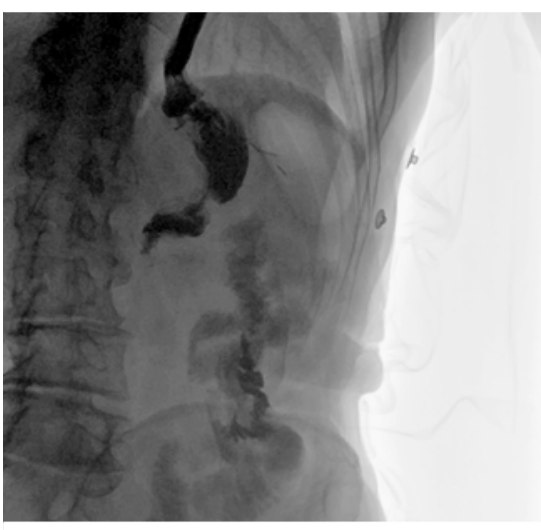

- Fig. 1 Radiographic image following oral contrast showing a normal esophagojejunal anastomosis after total gastrectomy, with a dilated jejunal limb due to malignant obstruction.

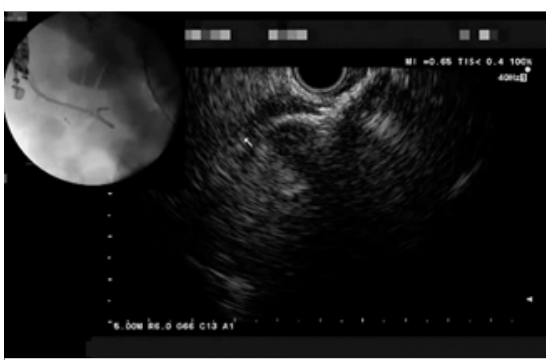

- Fig. 2 Endoscopic ultrasound image (inset: radiographic image) showing the deployment of the distal lumen-apposing metal stent (LAMS) flange.
Endoscopic ultrasound (EUS)-guided gastrointestinal anastomosis with a lumenapposing metal stent (LAMS) is an effective alternative to intraluminal stenting for upper gastrointestinal obstruction $[1,2]$. Recently, EUS-guided jejuno-jejunal anastomosis (EUS-JJA) has been reported for adhesive obstruction after gastric bypass and afferent loop syndrome [3-5].

A 75-year-old man presented with progressive epigastric pain and weight loss due to advanced malignancy after a previous total gastrectomy. Peritoneal recurrence had been diagnosed 2 years after the gastrectomy, and he had received chemotherapy, achieving stable disease for more than 3 years. On this presentation, as celiac trunk infiltration had been found on a recent computed tomography (CT) scan, it was agreed after multidisciplinary discussion that EUS-guided celiac plexus neurolysis should be performed; however, this failed to provide pain relief. At the time of EUS, a tight jejunal stenosis was observed $8 \mathrm{~cm}$ below the esophagojejunal anastomosis ( $\mathbf{F i g . 1}$ ).

Given the persistence of symptoms, together with vomiting, the patient underwent EUS-JJA 2 weeks later, after receiving a detailed explanation of the off-label use of the LAMS. A nasobiliary tube was advanced through the stricture and used to fill the jejunal limbs with a methylene blue-saline solution to look for an adequate operative window. A 19-gauge needle was then inserted into the target loop to check for methylene blue aspiration and to inject contrast. A $20 \times 10-\mathrm{mm}$ electrocautery-enhanced LAMS (HotAXIOS; Boston Scientific, Natick, Massachusetts, USA) was inserted "free-hand" and then dilated up to $18 \mathrm{~mm}$ ( $\triangleright$ Fig. 2 and Fig.3a,b). A pediatric gastroscope was finally advanced through the LAMS to assess the post-anastomotic jejunal limb ( $>$ Fig. $3 \mathbf{c}$ )

On the first postoperative day, a contrast study showed restoration of bowel transit ( $\triangleright$ Video 1 ) and oral feeding was resumed. The patient tolerated the procedure well and returned home 5 days later. He maintained adequate oral feeding and regained $2 \mathrm{~kg}$ of weight during the following 2 weeks, allowing chemotherapy to be restarted. Interestingly, he experienced significant improvement in pain control too.

EUS-JJA seems an effective treatment for malignant jejunal outlet obstruction.

\section{Endoscopy_UCTN_Code_TTT_1AS_2AG}
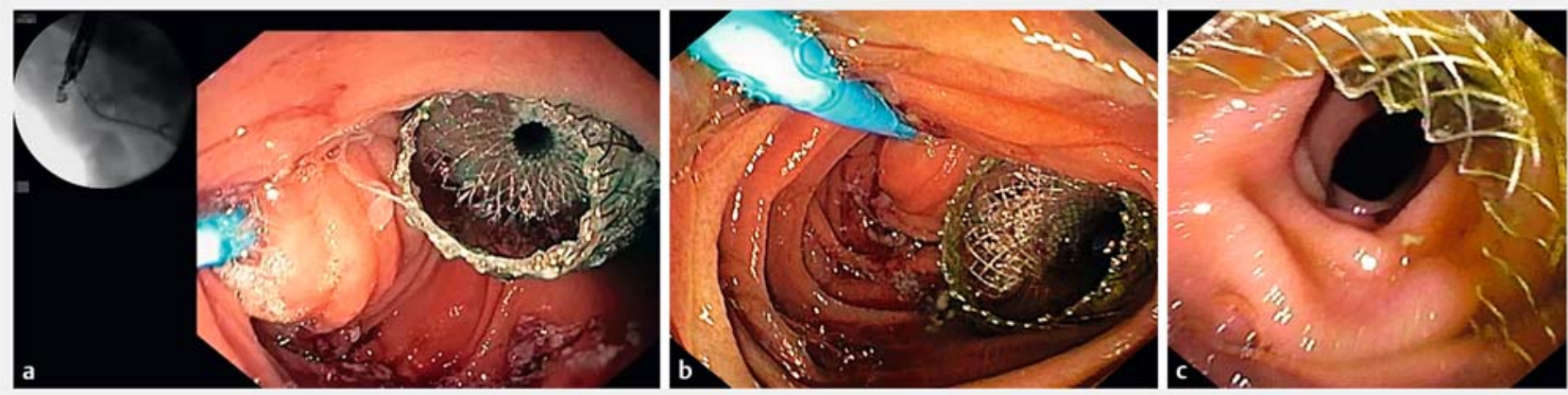

Fig. 3 Endoscopic images showing: a the released lumen-apposing metal stent (LAMS) adjacent to the nasobiliary tube that had been placed through the stricture (inset: radiographic image); $\mathbf{b}$ the endoscopic ultrasound-guided jejuno-jejunal anastomosis after dilation; $\mathbf{c}$ the jejunal limb viewed through the LAMS using a pediatric gastroscope. 


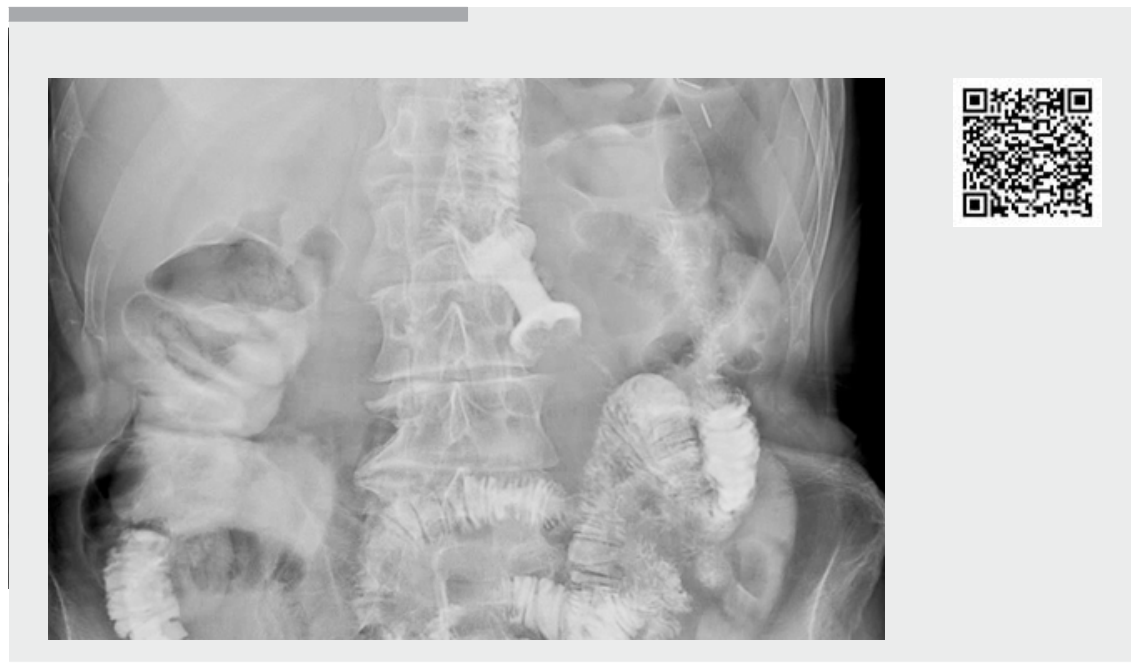

Video 1 Follow-up radiographic image 24 hours after the procedure showing normal passage of oral contrast medium through the jejuno-jejunal anastomosis, thereby confirming restored bowel transit.

\section{Competing interests}

The authors declare that they have no conflict of interest.

The authors

Andrea Lisotti ${ }^{1}$, Claudio Calvanese ${ }^{1}$, Martina Valgiusti $^{2}$, Igor Bacchilega ${ }^{3}$, Pietro Fusaroli ${ }^{1}$

1 Gastroenterology Unit, Hospital of Imola (BO), University of Bologna, Italy

2 Department of Medical Oncology, Istituto Scientifico Romagnolo per lo Studio e la Cura dei Tumori (IRST) IRCCS, Meldola (FC), Italy

3 Intensive Care Unit, Hospital of Imola (BO), University of Bologna, Italy

\section{Corresponding author}

\section{Andrea Lisotti, MD}

Gastroenterology Unit, Hospital of Imola, Department of Medical and Surgical Science, University of Bologna, Via Montericco 4, 40026 Imola (BO), Italy lisotti.andrea@gmail.com

\section{References}

[1] Fusaroli P, Jenssen C, Hocke M et al. EFSUMB Guidelines on Interventional Ultrasound (INVUS), Part V. EFSUMB Leitlinien Interventioneller Ultraschall (INVUS), Teil V. Ultraschall Med 2016; 37: 77-99

[2] Antonelli G, Kovacevic B, Karsensten JG et al. Endoscopic ultrasound-guided gastro-enteric anastomosis: A systematic review and meta-analysis. Dig Liver Dis 2020.

doi:10.1016/j.dld.2020.04.021

[3] Dioscoridi L, Forti E, Pugliese F et al. Buried lumen-apposing metal stent after gastrojejunal bypass. Endoscopy 2020; 52: E134E135

[4] Mutignani M, Forti E, Larghi A et al. Endoscopic entero-enteral bypass: an effective new approach to the treatment of postsurgical complications of hepaticojejunostomy. Endoscopy 2019; 51: 1146-1150

[5] Majmudar K, Wagh MS. EUS-guided jejunojejunostomy with lumen-apposing metal stent for complete jejunal obstruction after gastric bypass. Gastrointest Endosc 2016; 84: 853-854

\section{Bibliography}

Endoscopy 2021; 53: E232-E233

DOI 10.1055/a-1247-4391

ISSN 0013-726X

published online 23.9.2020

(C) 2020. Thieme. All rights reserved.

Georg Thieme Verlag KG, Rüdigerstraße 14,

70469 Stuttgart, Germany

\section{ENDOSCOPY E-VIDEOS}

https://eref.thieme.de/e-videos

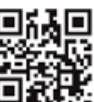

Endoscopy E-Videos is a free access online section, reporting on interesting cases and new

techniques in gastroenterological endoscopy. All papers include a high quality video and all contributions are freely accessible online.

This section has its own submission website at https://mc.manuscriptcentral.com/e-videos 